\title{
UNIQUE CONTINUATION FOR SCHRODINGER OPERATORS WITH POTENTIAL IN MORREY SPACES
}

\author{
ALBERTO RUIZ AND LUIS VEGA
}

\section{0 . Introduction}

Let us consider in a domain $\Omega$ of $\mathbf{R}^{n}$ solutions of the differential inequality

$$
|\Delta u(x)| \leq V(x)|u(x)|, x \in \Omega,
$$

where $V$ is a non smooth, positive potential.

We are interested in global unique continuation properties. That means that $u$ must be identically zero on $\Omega$ if it vanishes on an open subset of $\Omega$.

There is an extensive literature on the matter, mainiy to relax the local integrability condition required to the potential $V$. When $L_{l o c}^{p}$ classes are considered, $p \geq n / 2$ is a necessary and sufficient condition for the strong unique continuation property $[\mathbf{J K}]$ (see $[\mathbf{K}]$ for references). In this paper we shall consider some spaces introduced by Morrey [M], which have been recently used by C. Fefferman and D.H. Phong [FP] in studing the eigenvalues of Schrodinger operators; these spaces contain $L_{\text {loc }}^{n / 2}$.

We say that $V \in F^{p}, L_{p, \lambda}$ with $\lambda=2 p-n$ in classical notation $[\mathbf{P}]$, if

$$
\|V\|_{F_{\nabla}}=\sup _{Q}|Q|^{2 / n-p}\left(\int_{Q}|V|^{p}\right)^{1 / p}<\infty
$$

where the sup is taken over all cubes in $\mathbf{R}^{n}$ and $|Q|=$ Volume of $Q$. Notice $F^{p} \subset F^{q}$ if $p \geq q$.

In this paper we prove that any solution of (1) has the global unique continuation property if $V \in F_{l o c}^{p}$ and $p>(n-2) / 2$

Very recentiy $T$. Wolf has obtained the same result with a different approach.

We would like to thank C. Kenig for telling us about $T$. Wolf's result.

This improves the previously known results where $p>\frac{(n-1)}{2}$ (see [CS] and [ChR]).

The point to obtain this improvement is that in the above works the Carleman estimate is seen as a consequence of a uniform Sobolev inequality (see [KRS]).

$$
\|u\|_{L^{2}(V)} \leq C\|V\|_{F^{p}}\left\|\left(\Delta+a_{j} \partial / \partial x_{j}+b\right) u\right\|_{L^{2}(V-1)},
$$


where $C$ is independent of the linear perturbation of the Laplacian. Nevertheless, we prove directly the Carleman estimate

$$
\left\|e^{T x_{n}} u\right\|_{L^{2}(V)} \leq C\|V\|_{F P}\left\|e^{T x_{n}} \Delta u\right\|_{L^{2}\left(V^{-1}\right)},
$$

where $C$ is independent of $\tau$ for $\tau$ in $\left(\tau_{0}, \infty\right)$.

As we shall see while (2) is based on the restriction theorem for the Fourier Transform on the $(n-1)$-dimensional sphere, together with classical theory of weights, our proof follows from a detailed analysis of the multiplier associated to (3) which just involves the restriction theorem in dimension $\mathrm{n}-2$. Therefore the assumption in $p$ comes from the restriction operator in the sphere. We think that this is just a technical obstruction and the restriction theorem should be true for $p \geq 1$. Notice that we are close in the case $n=4$. We also remark that $F_{l o c}^{l}$ contains the so called Kato-Stummel class which B. Simon has conjectured is enough to assure unique continuation (see $[\mathbf{S}]$ ).

In the sequel we denote by $H_{\text {loc }}^{2}(\Omega)$ the classical Sobolev space, and

$$
A v_{Q} f=(1 /|Q|) \int_{Q} f
$$

We define the local Morrey class as the functions $W$ such that

$$
\|W\|=\sup _{y \in \Omega} \limsup \sup _{r \rightarrow 0}\left\|\chi_{B(y, r)}(.) W(.)\right\|_{F^{p}}<\infty .
$$

The main theorem is:

Theorem 1. Let $u \in H_{l o c}^{2}(\Omega), n \geq 3$, be a solution of (1), then there exists an $\varepsilon>0$, only depending on $p$ and $n$, such that if $V \in F_{l o c}^{p},\|V\|_{F p}\langle\varepsilon, p\rangle$ $(n-2) / 2$. and $u$ vanishes in an open subdomain of $\Omega$, then $u$ must be zero everywhere in $\Omega$.

The proof is related to a restriction theorem for the Fourier Transform, obtained in [CS] and [ChR], for which we are going to give an casy proof. Let us define, for this purpose, the Morrey classes; we say that $V$ is in $F^{\alpha, p}$ if

$$
\|\| V \mid \|_{\alpha, p}=\sup _{r, x} r^{\alpha}\left(A v_{B(x, r)} V^{p}\right)^{1 / p}<\infty,
$$

where the sup is taken on all the balls contained in $\Omega$. This notation corresponds to $\mathcal{E}^{-\alpha, p}$ in $[\mathrm{P}], 1 \leq \alpha \leq n / p$. Also $F^{2, p}=F^{p}$.

Theorem 2. Let $d \sigma$ be the uniform measure on the unit sphere $S^{n-1}$ in $\mathbf{R}^{n}$, and $(d \sigma) \wedge$ its Fourier transform, let $V \in F^{\alpha, p}, p>(n-1) / 2(\alpha-1)$, and consider the operator

$$
T f(x)=(d \sigma)^{\wedge} * f(x) .
$$


Then there exists a constant $C$ such that

$$
\|T f\|_{L^{2}(V)} \leq C\|\| V\|\|_{\alpha, p}\|f\|_{L^{2}(V-\imath)}
$$

for any $f$ in $C_{0}^{\infty}$.

It would be interesting to understand how this theorem is related to the one in $[\mathrm{V}]$ for mixed norm introduced by Rubio de Francia in the study of Bochner-Riesz operators [R].

\section{The Carleman estimate}

It is standard to obtain Theorem 1 as a consequence of the following Carleman estimate. This reduction can be seen in the case of $L^{2}$ weighted estimates in $[\mathrm{CS}]$ or $[\mathrm{ChR}]$.

Theorem (1.1). There exists a constant $C>0$ such that for $V$ in $F^{p}$, $p>(n-2) / 2$, the inequality

$$
\left\|e^{T x_{n}} u\right\|_{L^{2}(V)} \leq C\|V\|_{F^{p}}\left\|e^{T x_{n}} \Delta u\right\|_{L^{2}\left(V^{-1}\right)},
$$

holds for every $u$ in $C_{0}^{\infty}$ and $\tau$ in $\mathbf{R}$.

Proof: We can reduce to the case $\tau=1$ in the following way:

Take $f(x)=e^{r x_{n}} u(x)$, then (1.1) reduces to

$$
\|f\|_{L^{2}(V)} \leq C\|V\|_{F p}\left\|P_{r}(D) f\right\|_{L^{2}\left(V^{-1}\right)},
$$

where $P_{\tau}(D)$ has symbol $P_{\tau}(\xi)=|\xi|^{2}-\tau^{2}+i \tau \xi_{n}$.

The change of variable $f\left(\tau^{-1} x\right)=g(x)$ reduces (1.2) to

$$
\|g\|_{L^{2}\left(V_{r}\right)} \leq C\left\|V_{\tau}\right\|_{F \nu}\left\|P_{1}(D) g\right\|_{L^{2}\left(V_{\tau}^{-2}\right)},
$$

where $V_{r}(x)=V\left(\frac{x}{r}\right)$, since $\left\|V_{\tau}\right\|_{F^{p}} \leq \tau^{2}\|V\|_{F^{p}}$.

Consider the inverse operator given by the Fourier multiplier

$$
(T g)^{\wedge}(\xi)=\frac{1}{P_{1}(\xi)} g^{\wedge}(\xi)
$$

Our theorem reduces to prove that $T: L^{2}\left(V^{-1}\right) \rightarrow L^{2}(V)$ for $V$ in $F^{p}, p>$ $(n-2) / 2$.

We are going to use a decomposition of $T$ in the phase space. Consider first

$$
P_{1}(\xi)^{-1}=\left(\varphi_{1}(\xi)+\varphi_{2}(\xi)+\varphi_{3}(\xi)\right) P_{1}(\xi)^{-1}=\sum_{i=1}^{3} m_{i}(\xi)
$$


where $\varphi_{i}$ is in $C_{0}^{\infty}, i=1,2$; supp $\varphi_{1} \subset\{|\xi|<1 / 2\}, \varphi_{1} \equiv 1$ in $\{|\xi|<1 / 4\}$; $\operatorname{supp} \varphi_{3} \subset\{|\xi|>2\}, \varphi_{3} \equiv 1$ in $\{|\xi|>3\}$.

The Fourier multiplier corresponding to $m_{1}$ has a kernel rapidly decreasing and hence satisfies the inequality. For $m_{3}$ just observe that it behaves like $|\xi|^{-2}$ and by known results, see [FeP], satisfies the inequality for $V$ in $F^{p}$ with $p>1$.

We may decompose $m_{2}$ as a finite sum of operators the worst of which is given by the multiplier

$$
\tilde{m}(\xi)=p_{1}(\xi)^{-1} \psi_{1}\left(\left|\xi^{t}\right|^{2}-1\right) \psi_{2}\left(\xi_{n}\right)
$$

with $\xi^{\prime}=\left(\xi_{1}, \ldots, \xi_{n-1}\right)$, supp $\psi_{2} \subset[-1,1]$, supp $\psi_{1} \subset[-1 / 4,1 / 4], \psi_{1} \in C_{0}^{\infty}$.

Now we may write

$$
\tilde{m}(\xi)=\sum_{j=1}^{\infty} \tilde{m}_{j}(\xi)
$$

for $\tilde{m}_{j}(\xi) \equiv m_{\delta}(\xi)=a_{j}(\xi) \psi_{1}\left(\frac{\left|\xi^{\prime}\right|-1}{\delta}\right) \psi_{2}\left(\frac{\xi_{n}}{\delta}\right), \delta=2^{-j}$, with appropiate $a_{j}$ with $\delta^{-1}<\left|a_{j}\right|<2 \delta^{-1}$.

Hence we may reduce our inequality to the study of the operator $K_{\delta}$ given by a Fourier multiplier which has $L^{\infty}$ norm as $\delta^{-1}$ and is supported in the "torus" $\left|\xi^{t}\right|-1<2 \delta,\left|\xi_{n}\right|<\delta$. It is enough to prove:

Lemma. For $0<\delta<1 / 2$ and $T_{\delta}$ defined by

$$
\left(T_{\delta}\right)^{\wedge}(\xi)=m(\xi) f^{\wedge}(\xi)
$$

where

$$
m(\xi)=\varphi\left(\frac{1-\left|\xi^{\prime}\right|}{\delta}\right) \varphi\left(\frac{\xi_{n}}{\delta}\right), \operatorname{supp} \varphi \subset[-1,1], \varphi \in C_{0}^{\infty}
$$

the following inequalities hold:

(i) $\left(\int\left|T_{\delta} f\right|^{2} V\right)^{1 / 2} \leq C \delta|\log \delta|\|V\|_{F p_{0}}\left(\int|f|^{2} V^{-1}\right)^{1 / 2}, p_{0}=(n-2) / 2$.

$$
\left(\int\left|T_{\delta} f\right|^{2} V\right)^{1 / 2} \leq C \delta^{1+\varepsilon}\|V\|_{F^{\nabla}}\left(\int|f|^{2} V^{-1}\right)^{1 / 2}, \text { with } 0<\varepsilon<1-(n-2) / 2 p
$$

Proof: Let us call $K(x)=m^{\wedge}(x)$ and consider $\left\{\psi_{j}\right\}$ a smooth partition of unity

$$
1=\sum_{j=0}^{\infty} \psi_{j}, \operatorname{supp} \psi_{j} \subset\left(2^{j-1}, 2^{j+1}\right) j=1,2, \ldots
$$


Define $T_{j} f=K_{j} * f$, where $K_{j}(x)=\psi_{j}\left(\left|x^{\prime}\right|\right) K(x)$ and $x=\left(x^{\prime}, x_{\mathfrak{n}}\right) \in \mathbf{R}^{n-1} \times \mathbf{R}$. We shall obtain a good estimate for $K_{j}$ which will allow us to sum in $j$.

On one hand observe that a straightforward calculation gives $\left|m_{j}(\xi)\right|=$ $\left|\left(K_{j}\right)^{\wedge}(\xi)\right| \leq C \min \left\{2^{j} \delta, 1\right\}$ and, as a consequence,

$$
\left(\int\left|T_{j} f\right|^{2}\right)^{1 / 2} \leq C \min \left\{2^{j} \delta, 1\right\}\left(\int|f|^{2}\right)^{1 / 2} .
$$

On the other hand for any natural number $m$ there exists a constant $C_{m}$ such that

$$
\left|K_{j}(x)\right| \leq C_{m} \delta^{2} 2^{-j(n-2) / 2}\left(1+\delta \mid x_{n}\right)^{-m}\left(1+\delta 2^{j}\right)^{-m} .
$$

Consider first the case $0 \leq j \leq 1+[\log 1 / \delta]$. For $k \in \mathbf{Z}$ we define

$$
K_{j k}(x)=K_{j}(x) \cdot \chi_{\left[k \delta^{-1},(k+1) \delta-1\right]}\left(x_{n}\right) .
$$

Then

$$
\left|K_{j k}(x)\right| \leq C_{m} \delta^{2} 2^{-j(n-2) / 2}(1+|k|)^{-m} .
$$

Finally we can make in $\mathrm{R}^{n}$ a grid with paralellepipeds $\left\{Q_{\nu}\right\}$ such that the dimension of $Q_{\nu}$ are $2^{j} \times \ldots \times 2^{j} \times \delta^{-1}$.

Call $f_{\nu}=f \cdot \chi Q_{v}$. Then

$$
\begin{aligned}
& \int\left|K_{j k} * f\right|^{2} w=\int\left|K_{j k} * \sum_{\nu} f_{\nu}\right|^{2} w \\
& \leq C \sum_{\nu} \int\left|K_{j k} * f_{\nu}\right|^{2} w \\
& \leq C\left(\sup _{\nu} \int_{Q^{*} \nu} w\right) \sum_{\nu}\left\|K_{j k} * f_{\nu}\right\|_{L^{\infty}\left(Q^{*} \nu\right)}^{2},
\end{aligned}
$$

where $Q_{\nu}^{*}$ is a paraleliepiped with the same center as $Q_{\nu}$ and side ten times bigger than the sides of $Q_{\nu}$. By (1.4) and Young's inequality

$$
\begin{aligned}
& \left.\leq C_{m} \delta^{4} 2^{-j(n-2)}(1+|k|)^{-2 m}\left(\sup _{\nu} \int_{Q_{\nu} \nu} w\right) \sum_{\nu}\left(\int\left|f_{\nu}\right|\right)\right)^{2} \\
& \leq C_{m} \delta^{4} 2^{-j(n-2)}(1+|k|)^{-2 m}\left(\sup _{\nu} \int_{Q^{*} \nu} w\right)^{2} \int|f|^{2} w^{-1}
\end{aligned}
$$

Now observe that if $w=V^{p_{0}}$ and $V \in F^{p_{a}}$, then

$$
\sup _{\nu} \int_{Q^{*} \nu} w \leq C\left(2^{j} \delta\right)^{-1} 2^{2 j}\|V\|_{F_{p_{0}}}^{p_{0}} .
$$


Thus,

$$
\left(\int \mid K_{j} * f \|^{2} V^{p_{0}}\right)^{1 / 2} \leq C \delta 2^{-j(n-4) / 2}\|V\|_{F_{0}}^{p_{0}}\left(\int|f|^{2} V^{-p_{0}}\right)^{1 / 2} .
$$

Interpolation with (1.3) gives

$\left(\int\left|K_{j} * f\right|^{2} V\right)^{1 / 2} \leq C \delta\|V\|_{F D O}\left(\int|f|^{2} V^{-1}\right)^{1 / 2}$, if $0 \leq j \leq 1+[\log 1 / \delta]$.

In the case $j \geq 1+[\log 1 / \delta]$, let us define $K_{j k}$ as $K_{j}(x) \chi_{\left[k 2^{j},(k+1) 2 j\right]}\left(x_{n}\right)$, with $k \in Z$. Now for $j$ fixed we consider in $\mathbf{R}^{n}$ a grid of cubes of side $2^{j}$. Repeating the above process we obtain

$$
\begin{aligned}
\left(\int\left|K_{j} * f\right|^{2} V^{p_{0}}\right. & )^{1 / 2} \\
& \leq C \delta^{2(1-m)} 2^{-j((n-2) / 2+2 m-2)}\|V\|_{F^{p_{0}}}^{p_{0}}\left(\int|f|^{2} V^{-p_{0}}\right)^{1 / 2} .
\end{aligned}
$$

Again interpolation with (1.3) gives for $j \geq 1+[\log 1 / \delta]$

$$
\left(\int\left|K_{j} * f\right|^{2} V\right)^{1 / 2} \leq C 2^{-j}\|V\|_{F^{D 0}}\left(\int|f|^{2} V^{-1}\right)^{t / 2}
$$

Adding up in $j$ we prove (i).

In order to prove (ii) we proceed as follows:

Define $K_{j}(x)=\psi_{j}(\delta|x|) K(x)$, with $\psi_{j}$ as above $j=0,1, \ldots$ and the support of $K_{j} \subset B\left(0,2^{j+1} \delta^{-1}\right)$. Then fix $j$ and construct a grid of cubes $\left\{Q_{\nu}\right\}$ os side $2^{i} \delta^{-1}$. Then it is enough to prove the estimate for $f_{\nu}=f \cdot \chi_{Q_{\nu}}$.

Take $V \in F^{p}$ and $(n-2) / 2=p_{0}<p<\infty$, let us call $w=V^{p / p_{0}}$, then

$$
\left(\int\left|T_{j} f_{\nu}\right|^{2} w\right)^{1 / 2} \leq\left(\int_{Q *_{\nu}}\left|T_{j} f_{\nu}\right|^{2} w\right)^{1 / 2}=\left(\int\left|T_{j} f_{\nu}\right|^{2} w_{\nu}\right)^{1 / 2} \text {, where }
$$

$w_{\nu}=w_{\chi Q_{\nu_{\nu}}} ;$ then $w_{\nu} \in F^{p_{0}}$ and

$$
\left\|w_{\nu}\right\|_{F p_{0}} \leq C\|V\|_{F p}^{p / p_{0}}\left(2^{j} \delta^{-I}\right)^{2\left(1-p / p_{0}\right)} \text { and then by }(\mathrm{i})
$$

$$
\left(\int\left|T_{j} f_{\nu}\right|^{2} w\right)^{1 / 2} \leq C \delta|\log \delta|\left(2^{j} \delta^{-1}\right)^{2\left(1-p / p_{0}\right)} \mid \eta V \|_{F_{p}^{p}}^{p_{p}}\left(\int\left|f_{\nu}\right|^{2} w^{-1}\right)^{1 / 2} .
$$

But also

$$
\begin{aligned}
\left(\int\left|T_{j} f_{\nu}\right|^{2}\right)^{1 / 2} & \leq C\left(\int\left|f_{\nu}\right|^{2}\right)^{1 / 2}, \text { and by interpolation }\left(\int\left|T_{j} f_{\nu}\right|^{2} V\right)^{1 / 2} \\
& \leq C \delta^{2-p / p_{0}}|\log \delta|^{p_{0} / p_{2}-2 j\left(I-p_{0} / p\right)}\|V\|_{F_{p}}\left(\int\left|f_{\nu}\right|^{2} V^{-1}\right)^{1 / 2},
\end{aligned}
$$

and (ii) is proved. 


\section{The Restriction theorem}

We give the proof of theorem 2. Let us remak again that this theorem is contained in [CS] and [ChR], but the simplicity of our proof justifies to write it here.

Proof of theorem 2: It is known that

$$
K(x)=(d \sigma)^{\wedge}(x)=|x|^{-(n / 2-1)} J_{n / 2-1}(|x|),
$$

where $J_{\lambda}$ designs the Bessel function of order $\lambda$. Then decompose

$$
\begin{aligned}
K(x) & =\sum_{j=0}^{\infty} K_{j}(x) \text { with } \\
K_{j}(x) & =(d \sigma)^{\wedge}(x) \psi_{j}(|x|), \quad j=1,2, \ldots, \operatorname{supp} \psi_{j} \subset\left[2^{j-1}, 2^{j+1}\right] \\
K_{0}(x) & =(d \sigma)^{\wedge}(x) \psi(|x|), \operatorname{supp} \psi \subset[-1,1] .
\end{aligned}
$$

The classical P. Tomas, estimate for the Fourier Transform of $K_{j}(x)$ gives us the boundedness of $T_{j}=K_{j}$ from $L^{2}$ to $L^{2}$ with norm $2^{j}$.

We can repeat the argument in the proof of theorem 1 and obtain, for $w=V^{p}$,

$$
T_{j}: L^{2}\left(w^{-1}\right) \rightarrow L^{2}(w) \text { with norm bounded by } 2^{-j(n-1) / 2}\left(\sup _{Q_{\nu}} \int_{Q_{\nu}} w\right)
$$

where $Q_{\nu}$ is a cube in the grid in $\mathrm{R}^{n}$ of side $2^{j}$. Since $V \in F^{\alpha, p}$, we obtain

$$
\left\|T_{j}\right\|_{L^{2}(w-1) \rightarrow L^{2}(w)} \leq C 2^{j(n-\alpha p-(n-1) / 2)}\|V\|_{\alpha, p}^{p} .
$$

Interpolation gives

$$
\left\|T_{j}\right\|_{L^{2}(V-1)-L^{2}(V)} \leq C\left(2^{j}\right)^{\frac{\eta-1+2 p(1-a)}{2 p}}\|V\|_{\alpha, p},
$$

the sum is convergent if $p>\frac{n-1}{2(\alpha-1)}$.

It is an open question if the above operator send $L^{2}\left(V^{-1}\right)$ to $L^{2}(V)$ for $V$ in $F^{\alpha, p}, p<(n-1) / 2$. The answer to this question would be the corner stone to extend unique continuation properties to potential in $F^{p}$ for $p \leq(n-2) / 2$.

\section{References}

[CS] Chanillo, S AND SAWyer, E., Unique continuation for $\Delta+V$ and the $\mathrm{C}$. Fefferman-Phong Class (to appear).

[ChR] CHIARENZA, F AND RUIZ, A., Uniform $L^{2}$-weighted Sobolev inequatities, Proceeding AMS. 
[FP] Fefferman, C and Phong, D.H., Lower bounds for Schrodinger equations, Journees "Eq. aux Derivees Partielles". Saint Jean de Monts, Societe Mathematique de France, 1982.

[JK] JerIson, D AND KENIG, C., Unique Continuation and absence of possitive eigenvalues for Schrodinger operators, Ann. Math. 121 (1985), 463-494.

[K] KENIG, C., "Restriction theorems, Carleman Estimates, Uniform Sobolev Inequalities and Unique Continuation," In Harmonic Analysis and PDE. J. García-Cuerva (Ed.), Lecture Notes in Math., 1988.

[KRS] KenIG, C, RuIz, A. AND SogGE, C., Uniform Sobolev Inequalitics and unique continuation for second order constant coefficients differential equations, Duke Math. J. 55, 2 (1987), 329-347.

[M] MORREY, C.B., "Multiple integral problems in the calculus of variations and related topics," Un. of California Publ., 1943.

[R] Rubio de Francia, J.L., Transference Principles for radial multipliers, Duke Math. J. 58, 1 (1989), 1-19.

[P] PeEtre, J., On the theory of $\mathcal{L}_{p, \lambda}$ Spaces, Journal of Functional Analysis 4 (1969), 71-78.

[S] Simon, B., Schrodinger Semigroups, Bull. A.M.S. 7, 3 (1982), 447-526.

[T] TOMAS, P., A restriction thcorem for the Fourier Transform, Bull. A.M.S. 81 (1975), 477-478.

[V] VEGA, L., EI Multiplicador de Schrodinger. La función maximal y los operadores de restricción, Tesis doctoral, Universidad Autónoma de Madrid, 1987.

Alberto Ruiz: Departamento de Matemáticas

Universidad Autónoma de Madrid

28049 Madrid

SPAIN

Luis Vega: Department of Mathematics

University of Chicago

Chicago III

U.S.A. 